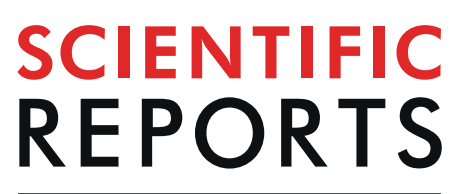

natureresearch

Check for updates

\title{
Tuning Penta-Graphene Electronic Properties Through Engineered Line Defects
}

\author{
Ramiro Marcelo dos Santos ${ }^{1 凶}$, Leonardo Evaristo de Sousa² ${ }^{2}$ Douglas Soares Galvão ${ }^{3}$ \& \\ Luiz Antonio Ribeiro Junior ${ }^{1}$
}

Penta-graphene is a quasi-two-dimensional carbon allotrope consisting of a pentagonal lattice in which both $s p^{2}$ and $s p^{3}$-like carbons are present. Unlike graphene, penta-graphene exhibits a nonzero bandgap, which opens the possibility of its use in optoelectronic applications. However, as the observed bandgap is large, gap tuning strategies such as doping are required. In this work, density functional theory calculations are used to determine the effects of the different number of line defects of substitutional nitrogen or silicon atoms on the penta-graphene electronic behavior. Our results show that this doping can induce semiconductor, semimetallic, or metallic behavior depending on the doping atom and targeted hybridization ( $s p^{2}$ or $s p^{3}$-like carbons). In particular, we observed that nitrogen doping of $s p^{2}$-like carbons atoms can produce a bandgap modulation between semimetallic and semiconductor behavior. These results show that engineering line defects can be an effective way to tune penta-graphene electronic behavior.

Research on 2D materials has gained much attention since the discovery of graphene ${ }^{1,2}$. This carbon allotrope that is composed of a hexagonal lattice was demonstrated to possess several exciting features, such as high electrical and thermal conductivity and large mechanical resistance that are aimed at developing the next generation of organic optoelectronic devices ${ }^{3,4}$. However, when it comes to optoelectronic applications, graphene has a zero bandgap, which precludes its use, for instance, as active material in solar cells. To overcome this issue, gap opening strategies have been developed ${ }^{5-7}$ including the cutting of graphene sheets into graphene nanoribbons ${ }^{8}$, the adsorption of $\mathrm{H}$-adatoms ${ }^{9}$ and the use of dopants ${ }^{10-12}$. Recently, the effects of such techniques have also been studied in similar 2D materials such as arsenen ${ }^{13,14}$, by means of first principle calculations.

When it comes to doping strategies, these are often divided into wet and dry doping methods ${ }^{15}$. The former makes use of the spin coating of dopant containing solutions so that charge transfer between graphene and dopant may take place. Dry doping strategies, on the other had, include the electrostatic field doping method, which consists on the application of an electric field perpendicular to the graphene sample. This method, however, cannot be applied to single layers of graphene. A more appropriate strategy in the context of this work is the atom substitution method, which can be performed by means of thermal treatment or plasma doping, for instance.

There are a few nature-occurring carbon allotropes, including graphite, fullerenes ${ }^{16}$, and carbon nanotubes ${ }^{17}$. Recently, another allotrope was proposed named penta-graphene ${ }^{18}$ (see Fig. 1), which has a lattice composed of pentagons that resemble the Cairo pentagonal tiling. It is supposed to present ultrahigh ideal strength even above that of graphene and to be able to withstand temperatures of up to $1000 \mathrm{~K}^{19}$. Penta-graphene was not synthesized yet, and it is believed that it can be at least a metastable material ${ }^{19-22}$. Furthermore, recent studies have suggested that chemical functionalization employing hydrogenation or fluorination could even increase its structural stability ${ }^{23,24}$. Electronic structure calculations indicate that penta-graphene is a semiconductor presenting bandgap values about 2.24-4.3 $\mathrm{eV}^{19,21,23}$. Such large bandgap suggests the need for gap tuning strategies to be developed in order to tailor this material for optoelectronic applications. In this sense, the particular topology of penta-graphene, which presents both $s p^{2}$ and $s p^{3}$-like carbon hybridizations in its lattice, raises the question of how its structural and electronic properties will behave by targeting a particular hybridization in the doping processes.

${ }^{1}$ Institute of Physics, University of Brasília, 70.919-970, Brasília, Brazil. ${ }^{2}$ Theoretical and Structural Chemistry Group, State University of Goias, 75133-050, Anapolis, Brazil. ${ }^{3}$ Applied Physics Department, State University of Campinas, Campinas, SP, 13083-959, Brazil. ${ }^{\circledR}$-mail: ribeirojr@unb.br 

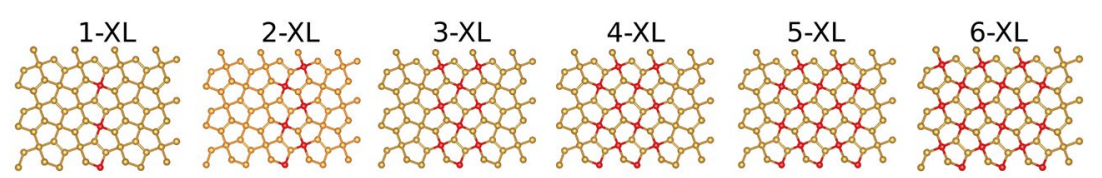

$7-X L$
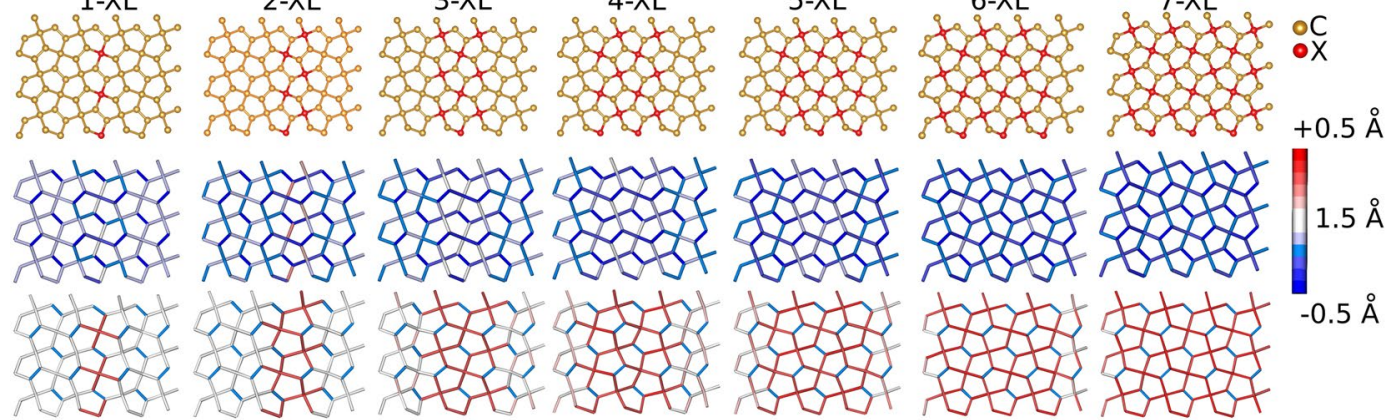

$1.5 \AA$

Figure 1. (Top) Schematic representation for the $s p^{3}$-like carbons doping strategy. The red color represents the doping atoms $(\mathrm{N}$ or $\mathrm{Si}$ ). Bond length variations for penta-graphene lattices with Nitrogen (Middle) and Silicon (Bottom) doping schemes. In the color palette on the right, the equilibrium distance is $1.575 \AA$. The maximum deviations are $0.5 \AA$ for Nitrogen and $1.5 \AA$ for Silicon doping schemes.

Herein, we carried out density functional theory (DFT) calculations to address the effects of the systematic substitutional doping of either $s p^{2}$ or $s p^{3}$-like carbons by nitrogen and silicon atoms in a penta-graphene lattice. Mainly, we investigate the effect of the different number of engineered line defects on their electronic and structural properties (see Fig. 1). As to the choice of dopants, size limitations require us to choose among atoms adjacent to carbon such as nitrogen, boron or silicon. In the particular case of nitrogen, it has been shown that nitrogen doping in graphene is responsible for regulating the electronic properties due to the ease with which nitrogen is able to control the local electronic structure, resulting in improvement of device performance ${ }^{25}$. Likewise, large area silicon doped graphene has been produced and has shown interesting properties ${ }^{26}$. Our findings show that, in terms of morphology, nitrogen doping is responsible for increasing the stiffness of the lattice in comparison to pristine penta-graphene, whereas silicon doping results in the simultaneous stretching and compression of $\mathrm{Si}-\mathrm{C}$ and $\mathrm{C}-\mathrm{C}$ bonds, respectively, concerning undoped $\mathrm{C}-\mathrm{C}$ bonds. The two doping schemes investigated here ( $\mathrm{Si}$ or $\mathrm{N}$ ) produce significantly different results in terms of electronic behavior. Silicon doping allows us to tune the bandgap when replacing $s p^{3}$-like carbons and produces metallic behavior when replacing $s p^{2}$-like ones. Nitrogen doping replacing $s p^{3}$-like carbons results in a transition from semiconductor to semimetallic to a metallic character. Nitrogen doping replacing $s p^{2}$-like carbons produces an alternating behavior between semimetallic and semiconductor depending on the number of dopants. These results indicate that engineered line defects can be a very effective way to tune penta-graphene electronic behavior.

\section{Results}

In the present work, we investigated the electronic and structural features of penta-graphene lattices with substitutional doping ( $\mathrm{N}$ or $\mathrm{Si}$ ) either at $s p^{3}$ or $s p^{2}$-like carbons forming engineered line defects. The cases of 1 up to 7 line defects were considered. In this sense, Fig. 1 (top panels) shows a schematic representation of these defective structures for the $s p^{3}$ case, which is similar to the $s p^{2}$ one. Figure 1 presents seven scenarios, which are identified by $\mathrm{N}-\mathrm{XL}$, with $\mathrm{N}$ corresponding the number of line defects in the horizontal/vertical directions, and $\mathrm{X}$ refers to either nitrogen or silicon dopant atoms.

Significant structural differences take place in the morphology of the resulting doped structures. For the nitrogen cases, an overall decrease in bond length values was observed, as presented in Fig. 1 (middle panels), where the blue color represents bond length compression when compared to the original non-doped ones. As expected, these effects are more pronounced around the defect lines, but they extend to the other bonds as the number of doping atoms increases. Such a reduction in bond length values can reach $0.5 \AA$, which corresponds to a roughly $30 \%$ decrease concerning the $1.575 \AA$ equilibrium distance found in pristine penta-graphene. In contrast, for the silicon atoms, the newly formed C-Si bonds undergo a substantial expansion of up to $1.5 \AA$, which would amount to $100 \%$ increase in bond length values when compared to pristine penta-graphene $\mathrm{C}-\mathrm{C}$ bonds, as shown in Fig. 1-bottom. For the remaining $\mathrm{C}-\mathrm{C}$ bonds, this effect is less pronounced.

It is well-known that structural modifications lead to changes in electronic properties. As such, useful information about these changes can be gained by contrasting the band structures of the doped and undoped penta-graphene sheets. Reports in the literature for penta-graphene indicate a semiconductor material with a bandgap of about $2.4 \mathrm{eV}^{18,21}$. We have obtained a similar value, as shown in Fig. 2 . In this figure, we also present the band results for the $s p^{3}$ cases for nitrogen (Fig. 2a) and silicon doping (Fig. 2b) as a function of the number of line defects. As we can see from Fig. 2, for the nitrogen with 1 line defect, the bandgap becomes indirect and decreases from $2.4 \mathrm{eV}$ to just $1.5 \mathrm{eV}$. The Fermi level lies near the conduction band, making this doped penta-graphene structure an n-type semiconductor. As the number of line defects increases, the material no longer presents a bandgap. We can see that for 2 and 3 defect lines, the partial density of states (PDOS) near the Fermi level, presented in Fig. S1a in the Electronic Supporting Information (SI), almost disappears. As the number of defect lines increases, the PDOS around the Fermi levels increases, and the doped penta-graphene becomes fully metallic.

The silicon doping of $s p^{3}$-like carbons, on the other hand, does not result in metallic materials. As seen in Figs. $2 \mathrm{~b}$ and S2a of the SI, doped penta-graphene lattices preserve their semiconductor nature. For the 1 line defect case, the only observed effect was the decrease in bandgap to $1.3 \mathrm{eV}$, with further doping making the 

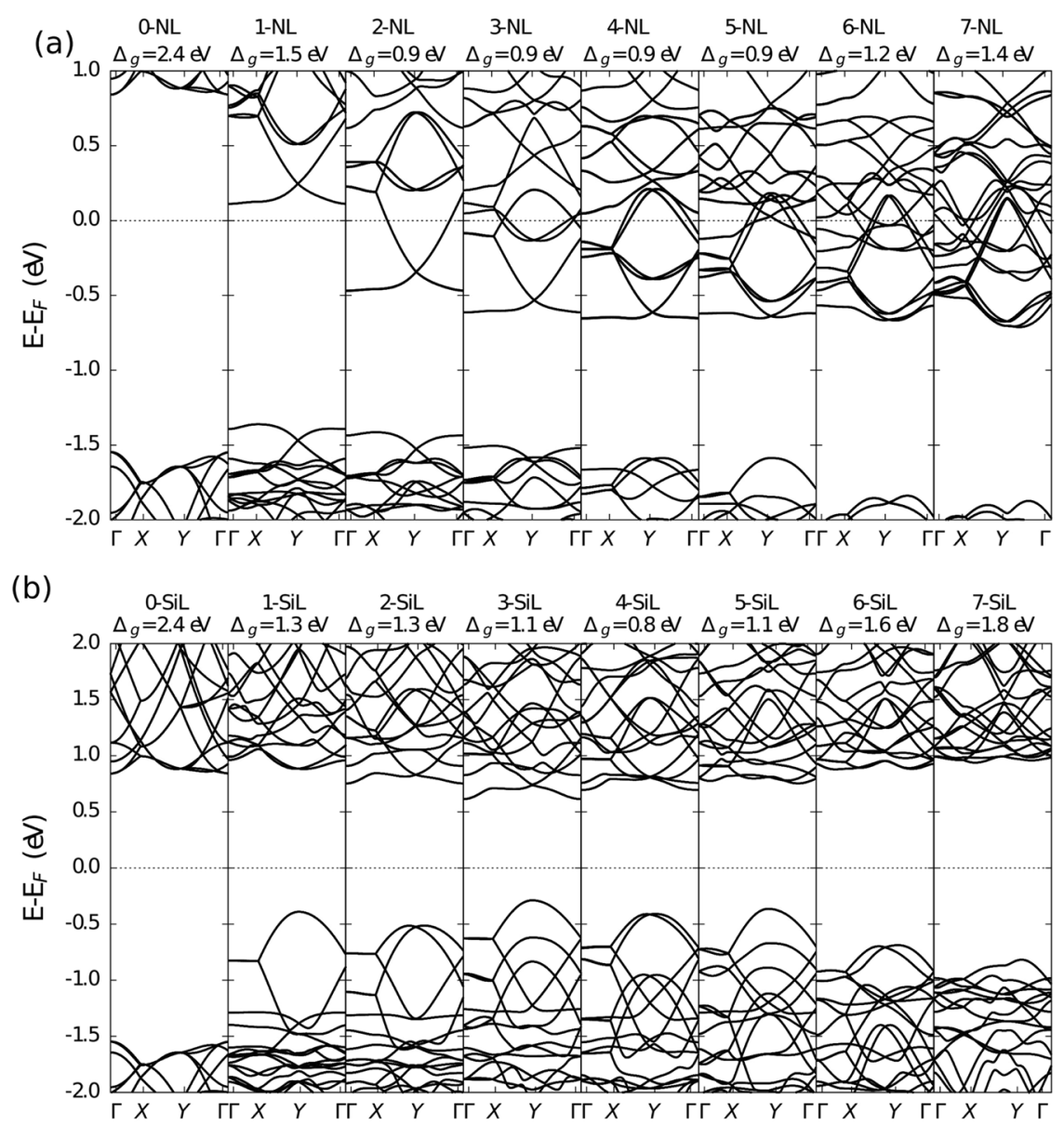

Figure 2. Band structures for undoped and doped penta-graphene lattices, as a function of the number of line defects for the $s p^{3}$ case. (a,b) correspond to the results for Nitrogen and Silicon doping schemes, respectively. Here, $\mathrm{X}-\mathrm{NL}$ and $\mathrm{X}$-SiL denote the number $(\mathrm{X})$ of dopant lines systematically inserted into the penta-graphene structure.

bandgap indirect. Interestingly, the bandgap dependence on the number of defect lines has a convex nature, with a minimum bandgap of $0.8 \mathrm{eV}$ observed for the case of 4 defect lines. After this point, the bandgap increases again, reaching $1.76 \mathrm{eV}$ for the lattice with seven defect lines. The decreases in bandgap values are associated with the appearance of states in the valence band closer to the Fermi level.

The second doping strategy considered here consists of the doping of $s p^{2}$-like carbons. One main difference is the possibility of having $\mathrm{N}-\mathrm{N}$ and $\mathrm{Si}-\mathrm{Si}$ bonds, not present for $s p^{3}$ case. The seven analyzed scenarios are presented in Fig. 3 (top panels). Again, nitrogen doping results in overall contraction of the bond length values up to $0.5 \AA$ concerning undoped penta-graphene ones. These deviations in the bond lengths are represented in Fig. 3 (middle panels) by the blue bonds. Silicon doping, in contrast, produces different patterns. These are mostly characterized by the simultaneous expansion of $\mathrm{Si}-\mathrm{C}$ bonds, by $\mathrm{Si}-\mathrm{Si}$ bonds that preserve the original $1.575 \AA$ bond length of pristine penta-graphene and by $\mathrm{C}-\mathrm{C}$ bonds that slightly contract. The combination of these effects results in the pattern characterized by blue hexagons diagonally sliced by red $\mathrm{Si}-\mathrm{C}$ bonds, as depicted in Fig. 3-bottom, especially for the six defect lines case. However, two particular cases, 5 and 7 defect lines, break the pattern producing much more disordered configurations likely induced by symmetry breaking. The $\mathrm{Si}-\mathrm{C}$ distances in the doped penta-graphene lattices can reach $3.8 \AA$, considerably larger than $\mathrm{Si}-\mathrm{C}$ bonds found, for instance, in disilicon carbide, which can be as large as $2.2 \AA^{27}$. This is suggestive that the atoms are no longer bonded and the structures undergo structural rearrangements.

In terms of electronic structure, nitrogen and silicon doping produce completely different results. Interestingly, for the $s p^{2}$ nitrogen doping cases, the bandgap values exhibit a bandgap modulation (alternating increasing/ decreasing) behavior, as can be seen in Fig. 4a. For even values of $N$, semiconducting properties are obtained with almost direct bandgaps that decrease as $N$ grows larger from $2.4 \mathrm{eV}$ for $\mathrm{N}=0$ to $0.6 \mathrm{eV}$ for $\mathrm{N}=6$. In contrast, for odd values of $N$, the valence band maximum (VBM) touches or surpasses the Fermi levels. However, the DOS near the Fermi level is very small, increasing progressively with $N$. This behavior can be better visualized in the PDOS plots of Fig. S1b in the SI. The even $N$ nitrogen-doped penta-graphene lattices display a semimetallic character, with carbon $p$ orbitals being mostly responsible for the DOS in the vicinity of the Fermi level.

Silicon doped penta-graphene structures, on the other hand, possess semiconductor properties only for $\mathrm{N}=$ 1 or $\mathrm{N}=2$, with a fast transition to bandgap closing. For larger $N$ values, the structures become fully metallic, as 


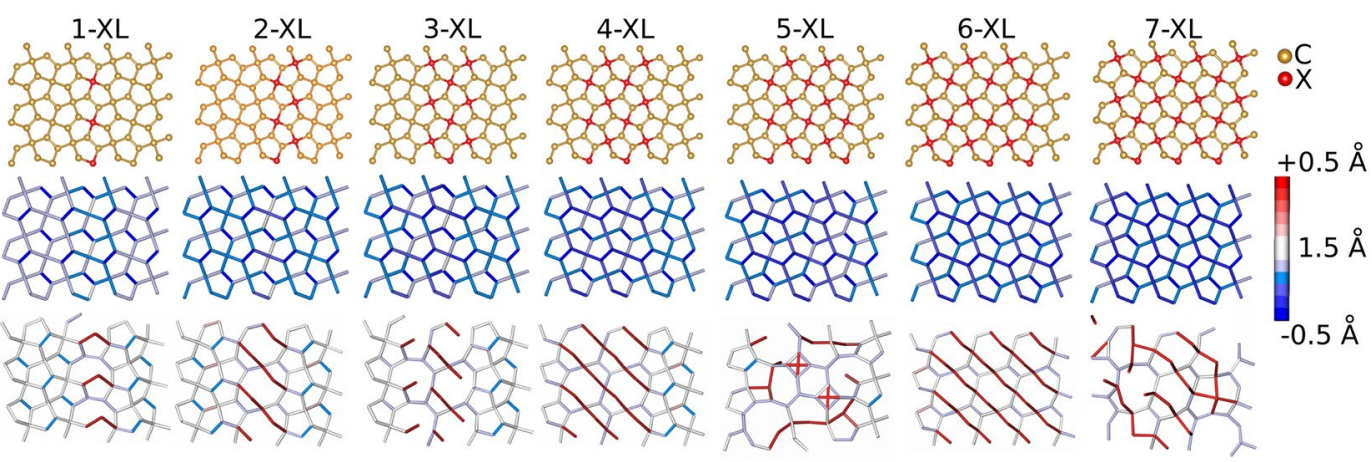

Figure 3. (Top) Schematic representation for penta-graphene lattices with $s p^{2}$-like carbons doping strategy. The red color represents the doping atoms $(\mathrm{N}$ or $\mathrm{Si})$. Bond length variations for penta-graphene lattices resulting from Nitrogen doping (Middle) and Similar Silicon doping scheme (Bottom). In the color palette on the right, the equilibrium distance is $1.575 \AA$. The maximum deviations are $0.5 \AA$ for Nitrogen and $1.5 \AA$ for Silicon doping schemes.
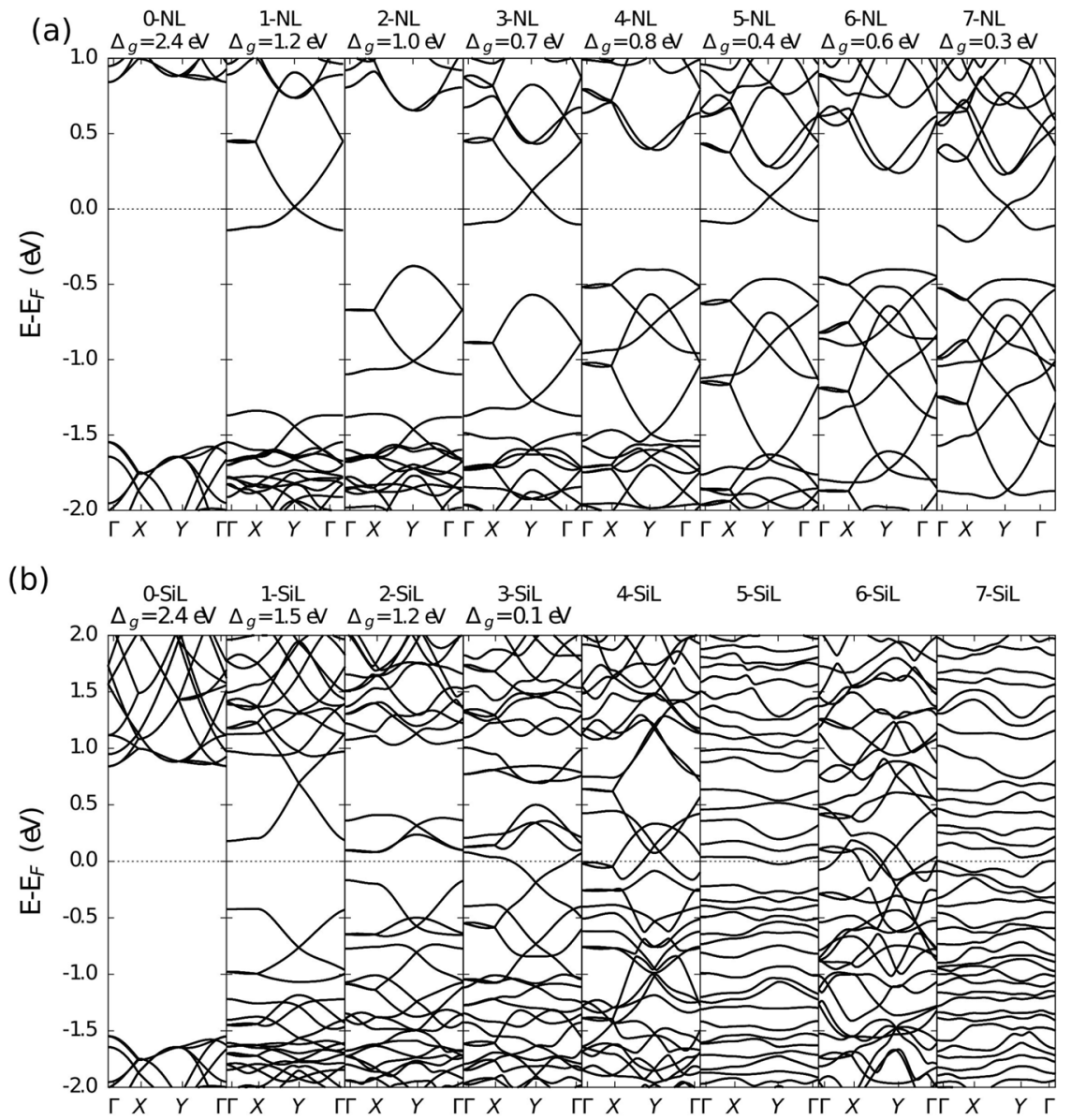

Figure 4. Band structures for undoped and doped penta-graphene, as a function of the number of line defects for the $s p^{2}$ case. $(\mathbf{a}, \mathbf{b})$ correspond to the results for Nitrogen and Silicon doping schemes, respectively. Here, $\mathrm{X}$-NL and X-SiL denote the number (X) of dopant lines systematically inserted into the penta-graphene structure.

shown in Fig. 4b (see also Fig. 2-ESI in SI). It is also worth noting that the effect of the disordered morphology observed for the 5-SiL and 7-SiL cases is to make energy levels practically independent of momentum when compared to the other cases, as evidenced by the almost flat (dispersionless) bands. In this sense, silicon doping of $s p^{2}$-like carbons is not as effective as in cases of nitrogen doping regarding decreasing the bandgap value.

An important advantage concerning the $s p^{2}$ doping instead of $s p^{3}$ one is the effects on the structural stability of the doped structures. In Fig. 5, we present the cohesive energy $\left(E_{c o h}\right)$ values for all doping scenarios. The cohesive 
(a)

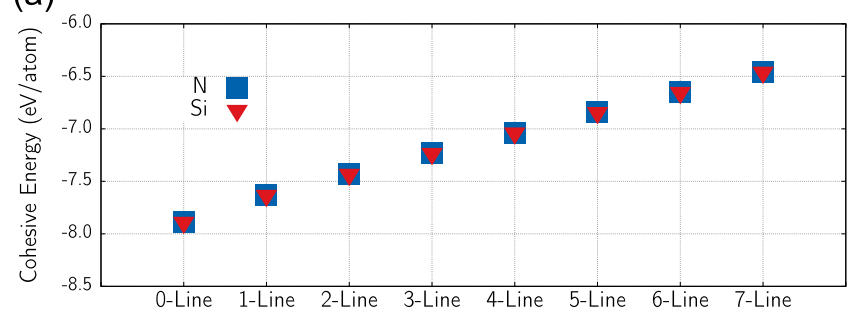

(b)

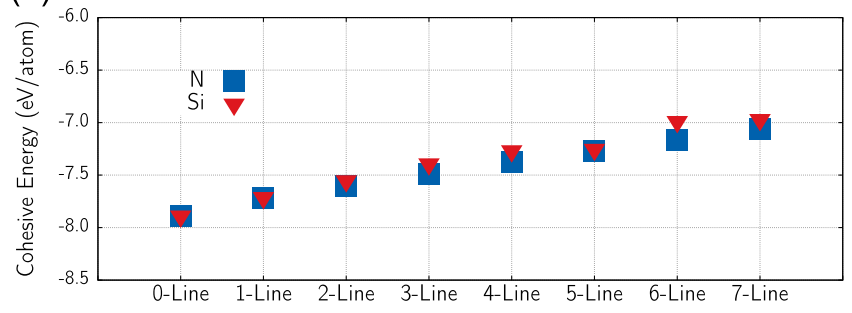

Figure 5. Cohesive energy for all cases of Nitrogen and Silicon doping schemes of (top) $s p^{3}$ carbons and (bottom) $s p^{2}$ carbons.

energy is the difference per atom between the entire system energy and the sum of the individual energies of its constituents. It is, therefore, associated with the stability of the system. Such a quantity is evaluated by the following expression:

$$
E_{\text {coh }}=\frac{\left(E_{\text {total }}-N_{D} E_{D}-N_{c} E_{C}\right)}{N_{\text {total }}},
$$

where $E_{\text {total }}$ is the total energy of the system. $N_{D}$ and $N_{C}$ are the number of dopant and carbon atoms of the system, respectively, and $N_{\text {total }}=N_{D}+N_{C}$. $E_{D}$ and $E_{C}$ are the energies of isolated dopant and and carbon atoms, respectively.

Figure 5-top) and Fig. 5-bottom) refer to $s p^{3}$ and $s p^{2}$ results, respectively. It can be seen from this figure that for both $s p^{3}$ and $s p^{2}$-like carbon doping, the effects on cohesive energy are similar for $\mathrm{N}$ and Si dopants. However, whereas $s p^{3}$-like carbon doping increases the cohesive energy from $-8.0 \mathrm{eV} /$ atom to $-6.5 \mathrm{eV} /$ atom, the corresponding increase in the case of $s p^{2}$-like carbon in less than $1.0 \mathrm{eV} /$ atom.

Finally, we present the charge transfer analysis for all the modeled systems studied here. Figure 6 illustrates the charge density profiles for all the cases of engineered line defects considering silicon (Fig. 6(a)) and nitrogen (Fig. 6(b)) dopants. For silicon-doped lattices, one can note that the charge density profile has an anti-bonding character in the doping regions for both sp2 and sp3 doping channels. As a consequence, charge density states have greater overlap in the regions that have only carbon atoms, as depicted in Fig. 6(a). Conversely, a metallic signature of the nitrogen-doped lattices can be inferred from the predominance of bonding states presented by the charge density localization, as shown in Fig. 6(b). Importantly, no substantial difference in the charge density profile is realized in nitrogen-doped lattices considering both sp2 and sp3 doping channels. In their electronic configuration, nitrogen possesses three electrons in the p orbital (last level 2p) whereas silicon has only two electrons in the last level (3p). The extra electron in the electronic arrangement of nitrogen leads to the greater overlap in the charge density profile and the formation of bonding states. The even (and smaller) number of electrons in the last level for silicon, when contrasted with nitrogen, is responsible for the smaller electronic correlation among the lattice sites, which considerably reduces the charge density overlap in the silicon-doped regions, as can be seen in Fig. 6(b).

\section{Methods}

DFT calculations were carried out within the Generalized Gradient Approximation (GGA) scheme as proposed for Perdew, Burke, and Ernzerhof $(\mathrm{GGA} / \mathrm{PBE})^{28}$ along with the $\mathrm{DZP}^{29,30}$, basis set. Relativistic pseudopotentials parameterized within the Troullier-Martins formalism were also considered ${ }^{31}$. These combined approximations can accurately describe the magnetic and electronic properties of materials composed of atoms with many electrons. All the calculations were performed considering spin polarization. For the bands and density of states calculations, Monkhorst pack grid of $21 \times 21 \times 3$ was used ${ }^{32}$. A mesh cutoff of 200 Ry was chosen as a parameter for our calculations ${ }^{33}$. The force criteria convergence was $0.001 \mathrm{eV} / \AA ̊$. In order to establish a good compromise between the accuracy of our results and computational costs, the tolerance in the matrix density and total energy was set to 0.0001 and $0.00001 \mathrm{eV}$, respectively. All calculations were performed with the SIESTA software suite ${ }^{34,35}$.

\section{Conclusions}

In summary, we have carried out DFT calculations to assess the changes in the structural and electronic properties of penta-graphene lattices resulting from selective $\mathrm{N}$ and Si doping (engineered line defects) of either $s p^{3}$ or $s p^{2}$-like carbons. Regardless of the type doping, for the nitrogen cases, we observed an overall stiffening of the penta-graphene structures. On the other hand, for the silicon doping cases, we observed only the stretching of $\mathrm{Si}-\mathrm{C}$ bonds and compression of the remaining $\mathrm{C}-\mathrm{C}$ bonds. 
(a)
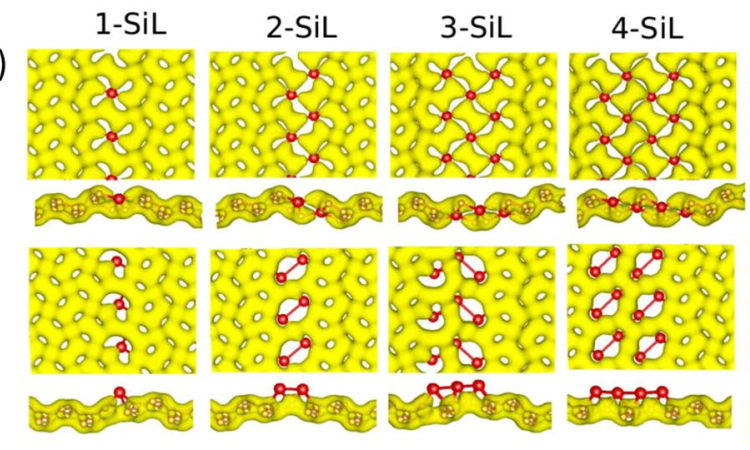

$1-\mathrm{NL}$

(b)

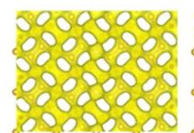

2-NL

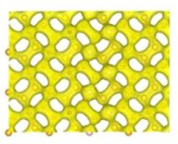

3-NL
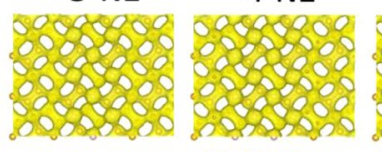

rgings
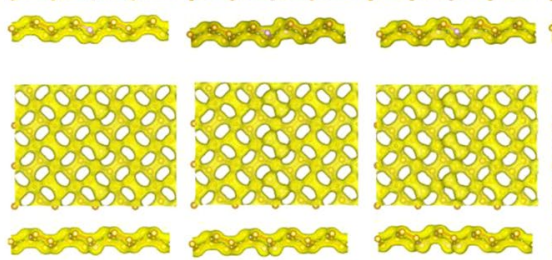

res?

aris?

ros
4-NL

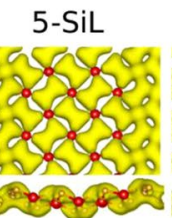

6-SiL

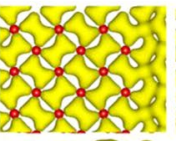

7-SiL
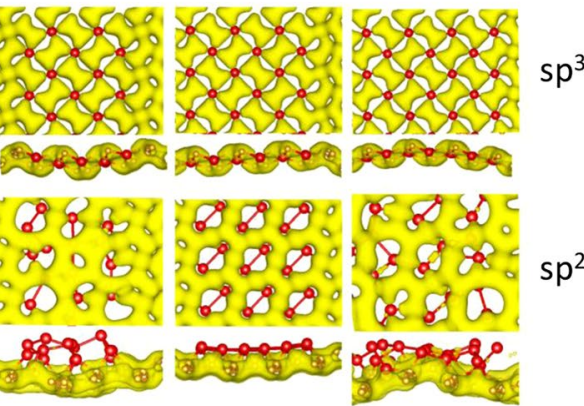

Pereares
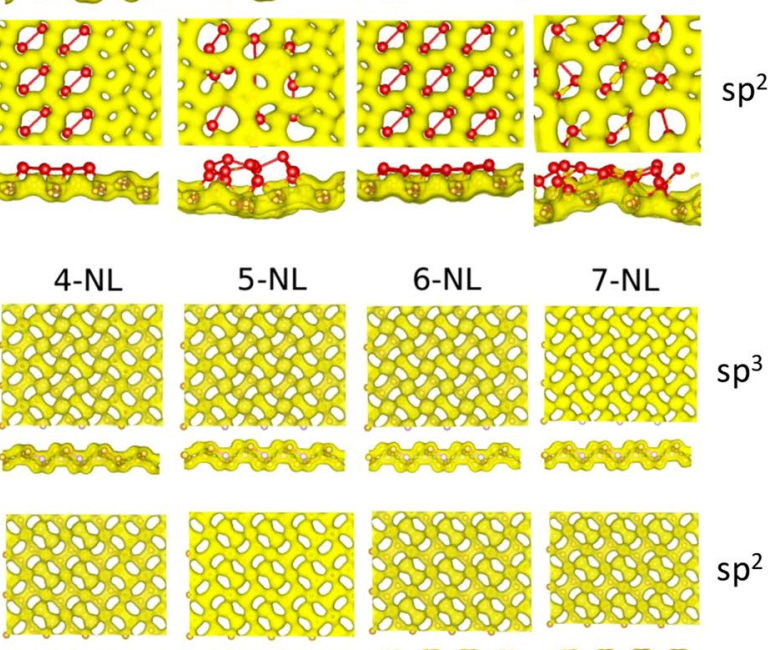

भर्तs?
$5-\mathrm{NL}$

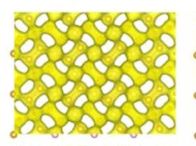

6-NL
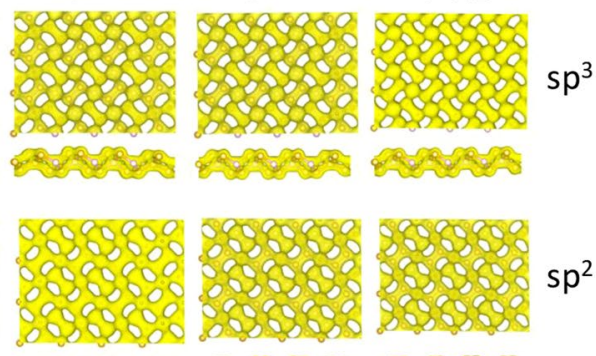

in: 189
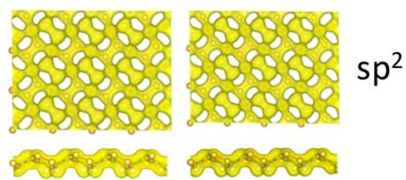

Figure 6. Charge density configuration for all the cases of engineered line defects presented here considering (a) silicon and (b) nitrogen dopants.

From an electronic structure perspective, both the doping type and doping atom selection produce significantly different results. Silicon doping of the $s p^{3}$-like carbons preserves the penta-graphene semiconductor character. The smallest observed bandgap value was $0.8 \mathrm{eV}$ (2.4 eV for pristine penta-graphene) for the 4 line defect case. For a larger number of line defects, the bandgaps increase again. On the other hand, for Si doping $s p^{2}$-like carbons, the result is a fast transition to metallic behavior.

Nitrogen doping produces more interesting results. For the $s p^{3}$-like carbons, the doping results in a progressive changes from semiconductor to metallic behavior. For the case of 1 line defects, the doped penta-graphene structure becomes an n-type semiconductor with a $1.5 \mathrm{eV}$ bandgap. For 2 and 3 line defects, the bandgap closes, but the density of states near the Fermi level goes to zero, giving the material a semi-metallic character. From this point on, further doping leads to true metallic behavior.

For the nitrogen doping of $s p^{2}$-like carbons, we observed a bandgap modulation behavior (alternating increase/decrease). For even numbers of line defects, the bandgap decreases with the number of line defects. In contrast, for odd numbers of line defects, a semi-metallic behavior is observed, combining zero bandgap with near-zero density of states at the vicinity of the Fermi level. Finally, the cohesive energy values indicate that doping the $s p^{2}$-like carbon affects less the structural stability of the resulting doped structures than $s p^{3}$-like carbon ones.

These results indicate that selective doping of penta-graphene structures through engineered line defects can be an effective tool to tune their electronic behavior, being possible to create structures that vary from large bandgaps through semiconductors and even metallic or semimetallic ones. Importantly, Liu and coworkers ${ }^{36}$ have demonstrated the creation of graphene and hexagonal boron nitride (h-BN) in-plane heterostructures with controlled domain sizes by using lithography patterning and sequential CVD growth steps. By employing this approach, the shapes of the graphene and h-BN domains were precisely controlled, and sharp graphene/h-BN interfaces were created. In this way, we believe that such an approach can be employed to yield our proposed model lattices. We hope the present work can stimulate further studies along these lines.

Received: 2 March 2020; Accepted: 9 April 2020;

Published online: 15 May 2020

\section{References}

1. Novoselov, K. S. et al. Electric field effect in atomically thin carbon films. Science 306, 666-669, https://doi.org/10.1126/ science.1102896 (2004).

2. Geim, A. K. \& Novoselov, K. S. The rise of graphene. Nature Materials 6, 183-191, https://doi.org/10.1038/nmat1849 (2007).

3. Neto, A. C., Guinea, F., Peres, N. M., Novoselov, K. S. \& Geim, A. K. The electronic properties of graphene. Reviews of modern physics 81, 109 (2009).

4. Balandin, A. A. et al. Superior thermal conductivity of single-layer graphene. Nano letters 8, 902-907 (2008).

5. Ilyasov, V. V., Meshil, B. C., Nguyen, V. C., Ershov, I. V. \& Nguyen, D. C. Tuning the band structure, magnetic and transport properties of the zigzag graphene nanoribbons/hexagonal boron nitride heterostructures by transverse electric field. J. Chem. Phy. 114, 014708, https://doi.org/10.1063/1.4885857 (2014). 
6. Shahrokhi, M. \& Leonard, C. Tuning the band gap and optical spectra of silicon-doped graphene: Many-body effects and excitonic states. Journal of Alloys and Compounds 693, 1185-1196, https://doi.org/10.1016/j.jallcom.2016.10.101 (2017).

7. Yankowitz, M. et al. Dynamic band-structure tuning of graphene moiré superlattices with pressure. Nature 557, 404-408, https:// doi.org/10.1038/s41586-018-0107-1 (2018).

8. Son, Y.-W., Cohen, M. L. \& Louie, S. G. Energy gaps in graphene nanoribbons. Physical review letters 97, 216803 (2006).

9. Zhou, Q., Yong, Y., Ju, W., Su, X. \& Li, X. Dft study of the electronic structure and magnetism of defective graphene decorated with hydrogen-adatom. Physica E: Low-dimensional Systems and Nanostructures 91, 65-71 (2017).

10. Denis, P. A. Band gap opening of monolayer and bilayer graphene doped with aluminium, silicon, phosphorus, and sulfur. Chemical Physics Letters 492, 251-257, https://doi.org/10.1016/j.cplett.2010.04.038 (2010).

11. Houmad, M., Zaari, H., Benyoussef, A., Kenz, A. E. \& Ez-Zahraouy, H. Optical conductivity enhancement and band gap opening with silicon doped graphene. Carbon 94, 1021-1027, https://doi.org/10.1016/j.carbon.2015.07.033 (2015).

12. Rani, P. \& Jindal, V. K. Study of $\mathrm{b}$ and $\mathrm{n}$ doped graphene by varying dopant positions. AIP Conference Proceedings 1512, 262-263, https://doi.org/10.1063/1.4791011 (2013).

13. Zhou, Q., Ju, W., Yong, Y. \& Li, X. Electronic and magnetic properties of 3d transition-metal atom adsorbed vacancy-defected arsenene: A first-principles study. Journal of Magnetism and Magnetic Materials 491, 165613 (2019).

14. Liu, Y., Zhou, Q., Ju, W., Li, J. \& Liu, Y. Influence of the vacancy-defect and transition-metal doping in arsenene: A first-principles study. Superlattices and Microstructures 132, 106163 (2019).

15. Oh, J. S., Kim, K. N. \& Yeom, G. Y. Graphene doping methods and device applications. Journal of nanoscience and nanotechnology 14, 1120-1133 (2014).

16. Kroto, H. W., Heath, J. R., O’Brien, S. C., Curl, R. F. \& Smalley, R. E. C60: Buckminsterfullerene. Nature 318, 162 (1985),

17. Iijima, S. \& Ichihashi, T. Single-shell carbon nanotubes of 1-nm diameter. nature 363, 603 (1993).

18. Zhang, S., Zhou, J., Wang, Q., Kawazoe, Y. \& Jena, P. Heterostructures based on two-dimensional layered materials and their potential applications. Proceedings of the National Academy of Sciences 112, 2372-2377 (2015).

19. Zhang, S. et al. Penta-graphene: A new carbon allotrope. Proceedings of the National Academy of Sciences 112, 2372-2377, https:// doi.org/10.1073/pnas.1416591112 (2015).

20. Cranford, S. W. When is 6 less than 5? penta-to hexa-graphene transition. Carbon 96, 421-428 (2016).

21. Rajbanshi, B., Sarkar, S., Mandal, B. \& Sarkar, P. Energetic and electronic structure of penta-graphene nanoribbons. Carbon 100, $118-125$ (2016).

22. Ewels, C. P. et al. Predicting experimentally stable allotropes: Instability of penta-graphene. Proceedings of the National Academy of Sciences 112, 15609-15612 (2015).

23. Einollahzadeh, H., Fazeli, S. M. \& Dariani, R. S. Studying the electronic and phononic structure of penta-graphane. Science and Technology of advanced MaTerials 17, 610-617 (2016).

24. Zhao, J. \& Zeng, H. Chemical functionalization of pentagermanene leads to stabilization and tunable electronic properties by external tensile strain. ACS Omega 2, 171-180, https://doi.org/10.1021/acsomega.6b00439 (2017).

25. Jeong, H. M. et al. Nitrogen-doped graphene for high-performance ultracapacitors and the importance of nitrogen-doped sites at basal planes. Nano letters 11, 2472-2477 (2011).

26. Lv, R. et al. Large-area si-doped graphene: controllable synthesis and enhanced molecular sensing. Advanced Materials 26, 7593-7599 (2014).

27. Koput, J. Ab initio potential energy surface and vibration-rotation energy levels of disilicon carbide, csi2. Journal of Molecular Spectroscopy 342, 83-91 (2017).

28. Perdew, J. P., Burke, K. \& Ernzerhof, M. Generalized gradient approximation made simple. Phys. Rev. Lett. 77, 3865-3868, https:// doi.org/10.1103/PhysRevLett.77.3865 (1996).

29. Hohenberg, P. \& Kohn, W. Inhomogeneous electron gas. Phys. Rev. 136, B864-B871, https://doi.org/10.1103/PhysRev.136.B864 (1964).

30. Kohn, W. \& Sham, L. J. Self-consistent equations including exchange and correlation effects. Phys. Rev. 140, A1133-A1138, https:// doi.org/10.1103/PhysRev.140.A1133 (1965).

31. Kleinman, L. \& Bylander, D. M. Efficacious form for model pseudopotentials. Phys. Rev. Lett. 48, 1425, https://doi.org/10.1103/ PhysRevLett.48.1425 (1982).

32. Monkhorst, H. J. \& Pack, J. D. Special points for brillouin-zone integrations. Phys. Rev. B 13, 5188, https://doi.org/10.1103/ PhysRevB.13.5188 (1976).

33. Anglada, E., M. Soler, J., Junquera, J. \& Artacho, E. Systematic generation of finite-range atomic basis sets for linear-scaling calculations. Phys. Rev. B 66, 205101, https://doi.org/10.1103/PhysRevB.66.205101 (2002).

34. Ordejón, P., Artacho, E. \& Soler, J. M. Self-consistent ujm order-n density-functional calculations for very large systems. Phys. Rev. B 53, 10441, https://doi.org/10.1103/PhysRevB.53.R10441 (1996).

35. Sánchez-Portal, D., Artacho, E. \&Soler, J. M. Density-functional method for very large systems with LCAO basis sets. Int. J. Quantum Chem. 65, 453 10.1002/(SICI) 1097-461X(1997)65:5<453::AID-QUA9>3.0.CO;2-V (1997).

36. Liu, Z. et al. In-plane heterostructures of graphene and hexagonal boron nitride with controlled domain sizes. Nature nanotechnology 8, $119(2013)$

\section{Acknowledgements}

The authors gratefully acknowledge the financial support from Brazilian Research Councils CNPq, CAPES, and FAP-DF, and CENAPAD-SP for providing the computational facilities. L.A.R.J. gratefully acknowledges the financial support from FAP-DF grant 00193.0000248/2019-32 and the financial support from CNPq grant 302236/2018-0.DSG thank the Center for Computational Engineering and Sciences at Unicamp for financial support through the FAPESP/CEPID Grant \#2013/08293-7.

\section{Author contributions}

R.M.S. and L.E.S. ran the calculations. R.M.S. and L.E.S. built the graphics. L.A.R. and D.S.G. interpreted the results and wrote the paper. All the authors were responsible for discussing the results.

\section{Competing interests}

The authors declare no competing interests.

\section{Additional information}

Supplementary information is available for this paper at https://doi.org/10.1038/s41598-020-64791-x.

Correspondence and requests for materials should be addressed to R.M.d.S. 
Reprints and permissions information is available at www.nature.com/reprints.

Publisher's note Springer Nature remains neutral with regard to jurisdictional claims in published maps and institutional affiliations.

(c) (i) Open Access This article is licensed under a Creative Commons Attribution 4.0 International License, which permits use, sharing, adaptation, distribution and reproduction in any medium or format, as long as you give appropriate credit to the original author(s) and the source, provide a link to the Creative Commons license, and indicate if changes were made. The images or other third party material in this article are included in the article's Creative Commons license, unless indicated otherwise in a credit line to the material. If material is not included in the article's Creative Commons license and your intended use is not permitted by statutory regulation or exceeds the permitted use, you will need to obtain permission directly from the copyright holder. To view a copy of this license, visit http://creativecommons.org/licenses/by/4.0/.

(C) The Author(s) 2020 